ORIGINAL ARTICLE

\title{
The role of biological maturation intervention and anthropometric factors on cardiac reserve index (OUES) in Iranian teenage boys
}

\section{Farzad Nazem*, Akbar Sazvar}

\author{
BU-ALI Sina University, Hamedan, Iran
}

Received 28 February 2015; accepted 25 May 2015

Available online 1 August 2015

\section{KEYWORDS \\ Maturity; Anthropometry; Oxygen uptake efficiency slope (OUES)}

\begin{abstract}
The development and organism change, especially changes in biological maturation process, is impressive on the physiological parameters in response to exercise. Hence, the new index oxygen uptake efficiency slope (OUES) role with biological maturity intervention and anthropometric factors of body weight, BSA and LBM which can measure the cardiorespiratory function in children maturity level normal and abnormal (early or late) with high health and safety is important. 72 healthy young males, with a mean age $13.95 \pm 1.8$ years and BMI $19.91 \pm 3.4 \mathrm{~kg} / \mathrm{m}^{2}$ were separated into two groups of normal puberty $(n=33)$ and abnormal maturation $(n=39)$. After consent obtaining, their $\mathrm{VO}_{2 \max }$ was determined by maximal aerobic test via gas analyzer $\left(\mathrm{VE}, \mathrm{VO}_{2}\right.$ and $\left.\mathrm{VCO}_{2}\right)$. The new index OUES was also determined according to logarithmic relationship Baba. A high correlation was observed between $\mathrm{VO}_{2 \max }$ and OUES physiological index $\left(R^{2}=0.90\right.$, SEE $\left.=292\right)$. There was no significant difference in the index OUES with and without the intervention of weight, BSA and LBM between normal and abnormal maturation of boys $(p>0.05)$. Significant correlation between $\mathrm{VO}_{2 \max }$ and normalized OUES with anthropometric variables was obtained $(R=0.83, R=0.87, R=0.91, p=0.00)$. OUES is influenced by weight, BSA and LBM. But the BSA role in the index of cardiac performance was more sensitive than the other two parameters. It appears that due to the lack of intervention maturation effect on this index, during the study of cardiorespiratory system physiological responses of children to exercise, regardless of the maturity the application of this index is possible.

(c) 2015 Consell Català de l'Esport. Generalitat de Catalunya. Published by Elsevier España, S.L.U. All rights reserved.
\end{abstract}

\footnotetext{
* Corresponding author.

E-mail addresses: farzadnazem2@gmail.com (F. Nazem), a_sazvar@Yahoo.com (A. Sazvar).
} 


\section{PALABRAS CLAVE}

Madurez; Antropometría; Gradiente de eficiencia de la captación de oxígeno (Oxygen Uptake Efficiency Slope (OUES))

\section{Introduction}

Maturity is a stage of physical development associated with the onset and progression of secondary sexual symptoms and is referred to as a symbol of the physiological functions of the body. Hence, the processes of development and progress in the assessment of general health is important.

Biological maturity events are different in communities as in addition to genetic factors, ethnic and geographical, social and economic factors and nutritional level influence the onset, delay the progress of its stages. ${ }^{2}$

Organism developmental changes during maturity can affect physiological factors such as cardiorespiratory fitness. As changes of practical capacity $\left(\mathrm{VO}_{2 \max }\right)$ in the development and evolution of the body are often influenced by fat free mass rather than body weight. In addition, the mean $\mathrm{VO}_{2 \max }$ per $\mathrm{kg}$ fat-free mass (FFM) decreases in the course of aging and after reaching maturity. Generally, in the process of organism development and growth, the practical capacity of cardiovascular system undergo some changes the same as other physical and physiological variables. But pattern of changes is not associated with sports activity intervention but depends on the quality of anatomical growth of organs and if the impact of such anthropometric interventions on the $\mathrm{VO}_{2 \max }$ (normalization) is not paid attention to by researchers, There appears the possibility of error results and non-real findings. ${ }^{3,4}$

On the other hand, measurement of respiratory gas exchange during exercise stress tests is an authoritative, efficient and non-invasive method to assess the efficiency of the cardiovascular system, respiratory physiology and metabolism of the active skeletal muscles of the body. In this context, maximal oxygen uptake $\left(\mathrm{VO}_{2 \max }\right)$ is the maximum mitochondrial volume of oxygen in low-stress myofibrils which are used in the maximum standard tests in sea level. ${ }^{5}$

$\mathrm{VO}_{2 \max }$ from a theoretical perspective is the point where the cost of active myofibril oxygen under load of specific job in the balance path between two scales of "the volume of oxygen required for oxidative metabolism and the consumed volume of $\mathrm{VO}_{2}$ during chemical mitochondrial reactions" reaches the intracellular physio-metabolic level so that even with increased exercise intensity, the amount of active oxygen myofibril is not added. ${ }^{6-8}$

However, it is a long time that $\mathrm{VO}_{2 \max }$ has been used a gold index to measure the level of cardiorespiratory fitness in different age groups and it is influenced by biological factors such as age, genetics, environmental factors and lifestyle, diet basket and body anthropometric dimensions or a combination of these variables.

Scientific evidence suggests the precise measurement of maximal oxygen uptake during maximal aerobic exercise 
test which is related to the individual maximum effort. It is possible that subjects, especially in the age range of the child or adolescent, cannot display high motivation and ultimately their ability to participate in the exercise protocols. Or in some patients with cardiovascular or respiratory failure, hypertension, renal failure and diabetes, who are faced with certain restrictions, selection and execution of the full maximum exhaustive tests is not safe and may be associated with risks. ${ }^{9}$

Therefore, it seems that immunization of sports protocols or clinical trials, achieving an efficient physiological index which can, without the need to maximal tests while considering anthropometric and biological maturity factors, providing reliable and accurate performance evaluation of cardiorespiratory system in the young individuals. In this direction, scientific reports introduce new criterion for measuring oxygen transfer performance called OUES or (slope between oxygen uptake and minute ventilation) in cardiorespiratory performance measurement, stronger and very accurate than $\mathrm{VO}_{2 \max } \cdot{ }^{10}$

OUES is a new index for evaluating the oxygen transfer system developed in 1996 by Japanese Dr Baba et al. ${ }^{11}$ and became interesting for biological science researchers. ${ }^{11-13}$ So that in 2010 Akkerman et al. ${ }^{14}$ wrote a review on OUES within 14 years of studies carried out, ${ }^{14}$ and in 2014 Buys et al. ${ }^{15}$ prepared norm for the index of OUES. This available scientific literature implies the importance of this new index.

OUES is the slope of the line between the two components of the oxygen cost $\left(\mathrm{VO}_{2}\right)$ and pulmonary ventilation (VE) during incremental ergometery on treadmill. Which represents an increase of oxygen consumption in response to minute ventilation increase in physical activity under specific workload and can be calculated from the linear relation $\mathrm{VO}_{2}=\log \mathrm{VE}+b$. In this formula, the slope (a) is an index of oxygen consumption or OUES. Doctor Baba converted the graph changes of $\Delta \mathrm{VO}_{2}, \Delta \mathrm{VE}$ into a linear relationship by calculating the logarithm of minute ventilation. Then, the slope of line between the $\mathrm{VO}_{2}$, Log VE was calculated and introduced it as a new index to measure cardiorespiratory performance or Oxygen Uptake Efficiency Slope (OUES). In this way, the correlation OUES was obtained in ratios of 90 and $75 \%$ of the execution time of maximal and exhaustive test at $R=0.96$ and $R=0.94$, respectively. ${ }^{11,12}$ In other words, in terms of submaximal aerobic work conditions, it is possible to measure the functional capacity of people with a high validity in parallel with a maximum protocol.

Previous studies indicate that OUES is highly reliable to measure cardiorespiratory function especially for children. In this regard, Rogowski et al., ${ }^{10}$ study in 2012 on the effects of biological maturation on OUES index of 8-27 year olds revealed that the index is influenced by the maturity meaning those who are not yet mature, have OUES lower than their adult peers. But when OUES is normalized and corrected in terms of body weight, the difference is reversed. And this was the only available study which investigated the effect of maturity on OUES of children. ${ }^{10}$

Also Akkerman et al. ${ }^{16}$ in 2010 on healthy children of 7-17 years old showed a high correlation of $R=0.95$ in both indices OUES and $\mathrm{VO}_{2 \max }$. But significant difference in the OUES index when performing two patterns of standard exhaustive and submaximal physical labor was not found and this was found that OUES is affected by anthropometric variables and thus it was suggested that OUES must be corrected by the intervention factors of BSA or FFM. ${ }^{16}$ Clinical study of Gadman et al. in 2008 on cardiac patients showed the validity of this index to measure the performance of cardiorespiratory system. ${ }^{17}$ Gruet et al. ${ }^{18}$ reported for pulmonary patients in $2010 R^{2}=0.83$ correlation between cardiorespiratory assessment scale: $\mathrm{VO}_{2 \max }$ and OUES at $80 \%$ time of maximal aerobic test of the patients.

Giardini et al. ${ }^{19}$ report in 2009 on young patients who had undergone open heart surgery revealed that the physiological findings of subjects in the second half of the maximal aerobic protocol i.e. in $50 \%$ of the ergometer protocol can be used in the clinical assessment of cardiorespiratory reserve. In this study, the correlation between the two indices OUES and $\mathrm{VO}_{2 \max }$ in these patients was $R=0.71 .{ }^{19}$ Other scientific evidence on the fat children ${ }^{20,21}$ or normal weight children ${ }^{22,23}$ also pointed out that the OUES can be used in the different ages to assess cardiorespiratory fitness level. Marinov's and Kostianev ${ }^{20}$ report on the influence of body composition on OUES showed that OUES In the obese children is lower than that in non-obese counterparts. But when the physiological scale was adjusted with body weight, OUES OF obese children was higher than that in the general population. ${ }^{20}$

Thus, it can be stated that taking advantage of the new index OUES in the terms of implementation of submaximal test and in shorter time, with the same reliability and sensitivity of the maximal aerobic standard test protocols, clinical response or safe threshold of physiological responses can be assessed under certain load.

According to the scientific literature in which the simultaneous impact of the maturity and body composition on index OUES has not taken into consideration. Therefore the aim of this study was to investigate the intervention of biological maturation in the adolescent boys with anthropometric variables of body weight, BSA and LBM on OUES index.

\section{Material and methods}

First, 4 schools (two elementary schools and two secondary schools) of the Hamadan area were randomly selected. Then, 72 healthy boys with age range of 11-17 years with consents and written permission from their parents and coaches volunteers participated in this study. The study protocol was approved by the ethics committee of Hamadan University of Medical Sciences \& Health Services. The subjects and their parents accustom with the objectives of the project. Subjects completed Health Questionnaire (PAR-Q) of American Association of Sports Medicine and the anthropometric characteristics with BMI percentiles ${ }^{24,25}$ were determined using the chart of American Centers for Disease Control and Prevention (CDC). The content of fat was obtained with the sum of two subcutaneous layers of the scapular and triceps based on regression relationship of Slaughter. ${ }^{26,27}$ The intensity of maximal aerobic test was obtained in two methods of heart rate reserve (HRR) by Karvonen relationship ${ }^{28}$ and the analog scale of psychosomatic feeling of work pressure by Borg (RPE) during the increasing implementation run of Graded Exercise Test (GXT). ${ }^{29}$ To measure the anthropometric parameters, the 
height, body mass were determined and to measure LBM, James WPT method was used. ${ }^{30,31}$ BSA was obtained by Haycock et al. ${ }^{32}$ relationship. To measure biological maturity in two early maturing and late maturing, Mirwald et al. ${ }^{33}$ method was used based on maturity offset and time to reach peak height velocity (PHV).

GXT protocol was executed on treadmill in Baba et al. ${ }^{11}$ method. This progressive standard sport program is designed in ten minutes for children in which children the speed and slope increase in accordance with exercise time increase and the individual continues to run beyond his lactate level on a treadmill equipped with automatic system of respiratory gas analyzer (Ganshorn - Germany). The criterion used to assess $\mathrm{VO}_{2 \max }$ included (1) a respiratory gains upper than unit (RER $>1.1$ ) according to $\Delta \mathrm{VCO}_{2} / \Delta \mathrm{VO}_{2}$ variation on system monitor, (2) when the heart rate reaches over $185 \mathrm{bpm}$ and (3) the feeling of body work pressure equaling to RPE > 17, $\left(\mathrm{RPE}_{20}\right)$ and the exhaustion announcement of real will. ${ }^{17,34}$ In this protocol, time for the first four stages of ergometry, $15 \mathrm{~s}$ and for stage 5-7 three minutes were considered. Finally, the total time of GXT protocol was completed in 10 min. ${ }^{11,12}$ To measure the $\mathrm{VO}_{2}$ in direct method of breathto-breath gas analyzer, the mean of each $10 \mathrm{~s}$ of measuring concentrations of $\mathrm{VO}_{2}, \mathrm{VCO}_{2}$ and $\mathrm{VE}$ were recorded in the computer and the related physiological information in the last $20 \mathrm{~s}$ of ergometry was used to determine the practical capacity of children $\left(\mathrm{VO}_{2 \max }\right)$. The heart rate during running on treadmill in each second was measured by Polar telemetry model $\mathrm{T}_{34}$ of Germany to the end of protocol and kept in the device memory. Then, the value of OUES was calculated from linear relationship OUES $=a$ Log VE $+b .{ }^{11}$ The cardiovascular variables under study were investigated after two or three hours passing the light lunch with any pastries, fruit or coffee while trainers and sports clothes were used at 4-6 o'clock. GXT test was carried out in work physiology laboratory of Bu-Ali Sina University at $19-21^{\circ} \mathrm{C}$, relative humidity $39-43 \%$ at $1860 \mathrm{~m}$ above sea level.

\section{Statistical method}

In order to analyze data, the SPSS statistical software Ver. 16 was used. The normal distributions of values of $\mathrm{VO}_{2}$ in subjects were specified with Shapiro-Wilk test in normal maturity and non-normal maturity groups. Then, independent $T$-test was used to ensure the impact of natural maturity and non-natural maturity on the index OUES in terms of selected anthropometric parameters. Linear regression relationship was used to determine the relationship of two physiologic indexes of OUES and $\mathrm{vo}_{2}$ in two simultaneous efficacy situations of anthropometry factor intervention and maturity level and without intervention. The information of descriptive statistics of variables was determined in terms of mean $\pm S D$. The alpha was established at $P \leq 0.05$ for all statistics.

\section{Results}

In estimating the biological maturity of 72 healthy youth boys 11-17 years old, 33 persons were located in normal maturity group and 39 persons in non-normal maturity group. The anthropometric and physiological features of children are presented in Table 1 . With respect to the exercise heart rate $(199.8 \pm 4.6)$ beats per minute, percentage of heart rate reserve $(94.68 \pm 3.81)$ and respiratory exchange ratio $\left(\mathrm{VCO}_{2} / \mathrm{VO}_{2}: 1.26 \pm 0.08\right)$, it can be said that the subjects have done maximal physical effort in the implementation of the protocol GXT in Baba et al. method. The mean value of relative peak oxygen consumption $\left(37.12 \pm 10 \mathrm{mil} \mathrm{min}^{-1} \mathrm{~kg}^{-1}\right)$ could indicate the actual maximum cardiovascular performance and can be used to validate OUES in children during safe maximal aerobic ergometry. In addition, the high correlation is observed between two physiological indexes of OUES and $\mathrm{VO}_{2 \max }$. As seen in Table 2, OUES (in anthropometry non-intervention or normalization) did not show any significant difference between boys of normal maturity and non-normal maturity. After OUES was normalized in terms of weight variables, BSA and LBM, the pattern of lack of difference between groups continued.

As with the relationship of values for two indexes OUES and $\mathrm{VO}_{2 \max }$ in absolute form (without normalization) and relative form or with normalization in terms of weight, LBM and BSA, regarding Tables 3 and 4, there is seen to be high correlation between these two physiological indexes with selected anthropometric variables.

Table 1 Anthropometric and physiological characteristics during increasing ergometry in adolescent boys.

\begin{tabular}{|c|c|c|c|}
\hline Variables & SEM & SD & Mean \\
\hline Weight (kg) & 1.55 & 13.15 & 51.64 \\
\hline Age (year) & 0.21 & 1.8 & 13.95 \\
\hline RPE (20) & 0.1 & 0.9 & 18.6 \\
\hline$\%$ body fat & 1.2 & 10.3 & 20.7 \\
\hline LBM (kg) & 1.1 & 9.3 & 43.31 \\
\hline $\operatorname{BSA}\left(\mathrm{m}^{2}\right)$ & 0.03 & 0.25 & 1.51 \\
\hline$\% \mathrm{BMI}$ & 3 & 2.55 & 40.7 \\
\hline $\mathrm{HR}_{\text {exercise }}(\mathrm{bp} / \mathrm{min})$ & 0.55 & 4.6 & 199.8 \\
\hline$\% \mathrm{HRR}$ & 0.45 & 3.8 & 94.7 \\
\hline RER: $\mathrm{VCO}_{2} / \mathrm{VO}_{2}$ & .009 & 0.08 & 1.26 \\
\hline $\mathrm{VO}_{2 \max }\left(\mathrm{l} \mathrm{min}^{-1}\right)$ & 0.1 & 0.83 & 1.96 \\
\hline $\mathrm{VO}_{2 \max }\left(\operatorname{mil~min}^{-1} \mathrm{~kg}^{-1}\right)$ & 1.18 & 10 & 37.12 \\
\hline OUES $_{\max }\left(\mathrm{VO}_{2} \mathrm{mil} \mathrm{min}^{-1}\right) / \log _{10} \mathrm{VE}\left(\mathrm{L} \mathrm{min}^{-1}\right)$ & 105.3 & 893.7 & 1663.2 \\
\hline
\end{tabular}


Table 2 Independent samples test for OUES and $\mathrm{VO}_{2 \max }$ in two groups normal and abnormal maturity.

\begin{tabular}{|c|c|c|c|c|}
\hline \multirow[t]{2}{*}{ Variables } & \multirow[t]{2}{*}{ Sig } & \multirow[t]{2}{*}{$t$} & \multicolumn{2}{|c|}{ Mean \pm SD } \\
\hline & & & Abnormal maturity & Normal maturity \\
\hline $\mathrm{VO}_{2 \max }(\mathrm{L} \min -1)$ & 0.21 & 1.26 & $1.85 \pm 0.74$ & $2.1 \pm 0.92$ \\
\hline $\mathrm{VO}_{2 \max }(\mathrm{BSA}):\left(\mathrm{mil} \mathrm{min}^{-1} \mathrm{~m}^{-2}\right)$ & 0.4 & 0.85 & $1230 \pm 370$ & $1315 \pm 467$ \\
\hline $\mathrm{VO}_{2 \max }(\mathrm{LBM}):\left(\mathrm{mil} \mathrm{min}^{-1} \mathrm{LBM} \mathrm{kg}^{-1}\right)$ & 0.78 & 0.28 & $37.43 \pm 10.7$ & $38.5 \pm 13.5$ \\
\hline $\mathrm{VO}_{2 \max }\left(\mathrm{mil} \mathrm{min}^{-1} \mathrm{~kg}^{-1}\right)$ & 0.8 & 0.25 & $36.8 \pm 9.67$ & $37.4 \pm 10.5$ \\
\hline OUES $_{\max }$ (no normalizes) $\left(\left[\right.\right.$ mil. $\left.\left.\min ^{-1} \mathrm{VO}_{2}\right] /\left[\mathrm{L} \mathrm{min}{ }^{-1} \mathrm{VE}\right]\right)$ & 0.12 & 1.6 & $1511 \pm 813$ & $1843 \pm 963$ \\
\hline OUES $_{\max }$ (body weight) $\left(\left[\mathrm{mil} \mathrm{kg}{ }^{-1} \mathrm{~min}^{-1} \mathrm{VO}_{2}\right] /\left[\mathrm{mil} \mathrm{kg}^{-1} \mathrm{~min}^{-1} \mathrm{VE}\right]\right)$ & 0.33 & 0.98 & $29.54 \pm 13.7$ & $32.7 \pm 13.2$ \\
\hline OUES $_{\max }(\mathrm{LBM})\left(\left[\mathrm{mil} \mathrm{kg}^{-1} \mathrm{~min}^{-1} \mathrm{VO}_{2}\right] /\left[\mathrm{mil} \mathrm{kg}^{-1} \mathrm{~min}^{-1} \mathrm{VE}\right]\right)$ & 0.23 & 1.21 & $34.8 \pm 14.7$ & $39.2 \pm 15.7$ \\
\hline OUES $_{\max }(\mathrm{BSA})\left(\left[\mathrm{mil} / \mathrm{m}^{2} / \mathrm{min} \mathrm{VO}_{2}\right] /\left[\mathrm{mil} / \mathrm{m}^{2} / \mathrm{min} \mathrm{VE}\right]\right)$ & 0.18 & 1.34 & $992 \pm 445$ & $1141 \pm 491$ \\
\hline
\end{tabular}

Table 3 Correlation between OUES and $\mathrm{VO}_{2 \max }$ with normalizes and no normalizes.

\begin{tabular}{|c|c|c|c|c|c|c|}
\hline \multirow[t]{3}{*}{ Variables } & \multicolumn{3}{|c|}{$\mathrm{VO}_{2 \max }\left(\mathrm{Lmin}^{-1}\right)$} & \multicolumn{3}{|c|}{$\mathrm{VO}_{2 \max }\left(\mathrm{milmin}^{-1} \mathrm{~kg}^{-1}\right)$} \\
\hline & \multicolumn{3}{|c|}{$R$} & \multicolumn{3}{|c|}{$R$} \\
\hline & $\begin{array}{l}\text { All } \\
\text { subject }\end{array}$ & $\begin{array}{l}\text { Normal } \\
\text { maturity }\end{array}$ & $\begin{array}{l}\text { Abnormal } \\
\text { maturity }\end{array}$ & $\begin{array}{l}\text { All } \\
\text { subject }\end{array}$ & $\begin{array}{l}\text { Normal } \\
\text { maturity }\end{array}$ & $\begin{array}{l}\text { Abnormal } \\
\text { maturity }\end{array}$ \\
\hline \multirow[t]{2}{*}{ OUES $_{\max }$ (no normalizes) } & 0.95 & 0.95 & 0.96 & 0.81 & 0.86 & 0.78 \\
\hline & $\mathrm{Sig}=0.00$ & $\mathrm{Sig}=0.00$ & Sig $=0.00$ & Sig $=0.00$ & Sig $=0.00$ & Sig $=0.00$ \\
\hline \multirow[t]{2}{*}{ OUES $_{\max }$ (body weight) } & 0.83 & 0.9 & 0.81 & 0.89 & 0.92 & 0.87 \\
\hline & $\operatorname{Sig}=0.00$ & $\mathrm{Sig}=0.00$ & Sig $=0.00$ & Sig $=0.00$ & Sig $=0.00$ & Sig $=0.00$ \\
\hline \multirow[t]{2}{*}{ OUES $_{\max }($ LBM) } & 0.87 & 0.9 & 0.89 & 0.87 & 0.88 & 0.88 \\
\hline & $\operatorname{Sig}=0.00$ & $\mathrm{Sig}=0.00$ & Sig $=0.00$ & Sig $=0.00$ & Sig $=0.00$ & Sig $=0.00$ \\
\hline \multirow[t]{2}{*}{ OUES $_{\max }(B S A)$} & 0.91 & 0.93 & 0.92 & 0.86 & 0.88 & 0.86 \\
\hline & $\operatorname{Sig}=0.00$ & $\mathrm{Sig}=0.00$ & Sig $=0.00$ & Sig $=0.00$ & Sig $=0.00$ & $\mathrm{Sig}=0.00$ \\
\hline
\end{tabular}

\section{Discussion}

In this research, regarding the importance of OUES in assessment of respiratory-cardiac system efficiency in different age ranges and different individuals, the effect of anthropometric variables such as body weight, BSA, and LBM on OUES and child's organism growth process in maturity, the effect of maturity on OUES in adolescent boys in normal and non-normal maturity was evaluated. As seen in Table 2, the absolute mean of OUES (without normalization) at 1843 ([mil. $\left.\mathrm{min}^{-1} \mathrm{VO}_{2}\right] /\left[\mathrm{L} \mathrm{min}^{-1} \mathrm{VE}\right]$ ) in boys of normal maturity was greater than that in their peers of non-normal maturity, yet not significant. In another word, the maturity level (early mature, late mature) does not influence OUES index significantly. When OUES was normalized in terms of selected anthropometric variables, we got the same results.

It is worth mentioning that there was not found no scientific literature on the effect of normal and non-normal

Table 4 Correlation between OUES and $\mathrm{VO}_{2 \max }$ with normalizes and no normalizes.

\begin{tabular}{|c|c|c|c|c|c|c|}
\hline \multirow[t]{3}{*}{ Variables } & \multicolumn{3}{|c|}{$\mathrm{VO}_{2 \max }(\mathrm{BSA}):\left(\mathrm{mil} \mathrm{min}^{-1} \mathrm{~m}^{-2}\right)$} & \multicolumn{3}{|c|}{$\mathrm{VO}_{2 \max }(\mathrm{LBM}):\left(\mathrm{mil} \mathrm{min}^{-1} \mathrm{LBM} \mathrm{kg}^{-1}\right)$} \\
\hline & \multicolumn{3}{|c|}{$R$} & \multicolumn{3}{|c|}{$R$} \\
\hline & All subject & $\begin{array}{l}\text { Normal } \\
\text { maturity }\end{array}$ & $\begin{array}{l}\text { Abnormal } \\
\text { maturity }\end{array}$ & All subject & $\begin{array}{l}\text { Normal } \\
\text { maturity }\end{array}$ & $\begin{array}{l}\text { Abnormal } \\
\text { maturity }\end{array}$ \\
\hline \multirow[t]{2}{*}{ OUES $_{\max }$ (no normalizes) } & 0.88 & 0.86 & 0.9 & 0.71 & 0.72 & 0.71 \\
\hline & Sig $=0.00$ & Sig $=0.00$ & Sig $=0.00$ & Sig $=0.00$ & Sig $=0.00$ & Sig $=0.00$ \\
\hline \multirow[t]{2}{*}{ OUES $_{\max }$ (body weight) } & 0.87 & 0.88 & 0.88 & 0.81 & 0.72 & 0.84 \\
\hline & Sig $=0.00$ & Sig $=0.00$ & Sig $=0.00$ & Sig $=0.00$ & Sig $=0.00$ & $\mathrm{Sig}=0.00$ \\
\hline \multirow[t]{2}{*}{ OUES $_{\max }($ LBM) } & 0.91 & 0.9 & 0.93 & 0.84 & 0.85 & 0.85 \\
\hline & Sig $=0.00$ & Sig $=0.00$ & Sig $=0.00$ & Sig $=0.00$ & Sig $=0.00$ & $\mathrm{Sig}=0.00$ \\
\hline \multirow[t]{2}{*}{ OUES $_{\max }(\mathrm{BSA})$} & 0.91 & 0.9 & 0.93 & 0.81 & 0.82 & 0.82 \\
\hline & Sig $=0.00$ & Sig $=0.00$ & Sig $=0.00$ & Sig $=0.00$ & Sig $=0.00$ & Sig $=0.00$ \\
\hline
\end{tabular}


maturity on OUES but the study of Anthony et al. on healthy 8-27 year-old individuals in four groups before maturity, mid-maturity, after maturity and adult reported a significant difference between absolute value of OUES in groups of before maturity and mid-maturity. Of course, when relative value of OUES was normalized in terms of body weight and FFM, the result was reversed. ${ }^{10}$ They did not investigate the effect of early maturity and late maturity, but the general result of Rogowski et al.'s study was the effect of maturity on OUES which is not consistent with this paper. This difference can be due to division of individuals in four age groups in Rogowski et al.'s study rather than maturity level. As age increase enhances the relative values of OUES, the result of the previous study was probably dependent on this factor.

According to Tables 2 and 3 , when $\mathrm{VO}_{2 \max }$ is normalized to body weight, $\mathrm{VO}_{2 \max }$ correlation with OUES reached from $R=0.95$ to $R=0.81$. This reduction of correlation can be seen in both groups of normal and non-normal maturity after being corrected with body weight which is consistent with the study of Sun et al. ${ }^{23}$ They reported the drop of correlation between the physiological indices having been normalized with weight of healthy subjects aged $17-18$ years from $R=0.95$ to $R=0.76$. This result showed that OUES is being influenced by body weight component in the age range of the children to the elderly. ${ }^{23}$ In studies of Baba et al. and Akkerman et al. on children the same result was reported as the weight factor has a significant effect on OUES index. ${ }^{11,17}$

On the other hand, Marinov and Kostianev's study in 2003 , with an emphasis on body composition effect on obese and non-obese children revealed that absolute value of OUES in obese children is much lower than that in normal counterparts; in contrast, when normalized to body weight, the relative value of OUES in obese children was reported to be higher than non-obese children. ${ }^{20}$

In the study of Van Laytem in 2005 and 2006 on the cardiac patient, not only did the OUES correlation with $\mathrm{VO}_{2 \max }$ after being normalized to body weight not decrease but also increased from $R=0.73$ to $R=0.83 .{ }^{35,36}$ Possible cause of different result in this clinical study with our findings is due to the low level of practical capacity of cardiac patients while performing the exercise test.

In the present study, when both of $\mathrm{VO}_{2 \max }$ and OUES indexes in terms of body weight were normalized, the degree of correlation between the two indices have been increased slightly to $R=0.89$, which is likely to affect OUES more by body weight compared to other physiological index. In the effect on OUES by other anthropometric variables, the role of BSA and LBM interventions was also noted.

As can be seen in Tables 2-4, in two groups of boys with natural and non-natural maturity, a significant difference between the relative value OUES and $\mathrm{VO}_{2 \max }$ in terms of BSA and LBM was not found. But there was seen a high correlation with the relative $\mathrm{VO}_{2 \max }$ values in both groups. However, most correlation was observed between OUES normalized with the BSA and $\mathrm{VO}_{2 \max }$ (BSA) in males at natural maturity $(R=0.93)$, which shows the anthropometric variables can have a similar effect on OUES index.

Consistent with our results, Marinov et al. ${ }^{37}$ in 2007 obtained a lower correlation between the indices OUES and $\mathrm{VO}_{2 \max }$ after being normalized by BSA from $R^{2}=0.88$ to $R^{2}=0.76 .{ }^{37}$ Bongers et al.'s clinical assessment in 2011 obtained the correlation of OUES and $\mathrm{VO}_{2 \max }$ after being normalized by BSA in healthy children at $R=0.78$ and in children with cystic fibrosis patient at $R=0.54 .^{38}$

In this context, Akkerman et al. ${ }^{14}$ in 2010 indicated that in the review of the scientific literature, a high correlation between OUES and $\mathrm{VO}_{2 \max }$ after being normalized by BSA and LBM can be seen, which are consistent with the results obtained in Iranian adolescent boys. Drinkard et al.'s study on 12-17 year-old children in 2008 on the impact of body form, revealed that OUES is lower in obese children than non-obese children. But when OUES was moderated by the anthropometric factor of LBM, OUES in obesity children was significantly lower than non-obese counterparts. ${ }^{39}$

\section{Conclusion}

In summary, based on the results of this research, it can be said OUES is influenced by anthropometric variables selected. In other words, a significant association between $\mathrm{VO}_{2 \max }$ and OUES index normalized to body weight, LBM and BSA was obtained $(R=0.83, R=0.87, R=0.91)$. But it should be noted that the BSA parameter in the index of cardiac reserve performance is more sensitive than the other two parameters. So it seems, because of the lack of influence of adult intervention on the mentioned index in the study of the physiological responses of children's cardiorespiratory system to exercise, the application of this indicator is possible regardless of maturity.

\section{Limitations}

Limitations of this study include the motivational level of children to run exhaustive protocols, lack of measurement of arterial blood oxygen saturation during ergometry test and of the application of the method to determine the maturation level of subjects, and the possibly the sample size studied and selection style of voluntarily subjects.

\section{Conflict of interest}

The authors declare that they don't have any conflict of interests.

\section{Acknowledgements}

This article is adapted from Project of doctorate in exercise physiology by fellowship funds (research deputy of Bu-Ali Sina University). The authors express their appreciation to the staff of the department of education, students, parents and school managers of Hamedan city, who helped us in doing this research.

\section{References}

1. Giddens H, Wang L, Koch G. Secondary sexual characteristics in boys. Arch Pediatr Adolesc Med. 2001;155:1022-8.

2. MArshall WA, Tanner JM. Variations in pattern of pubertal changes in boys. Arch Dis Child. 1970;45:13-23.

3. Rowland T. Childeren's exercise physiology. 2nd ed. Human Kinetics; 2005. 
4. Malina R, Bouchard C, Bar-Or O. Growth, maturation, and physical activity. 2nd ed. Human Kinetics; 2004.

5. Kraemer w, Fleck SJ, Deschenes R. Exercise physiology integrating theory and application. 11th ed. Lippincott Williams \& Wilkins, a Wolters Kluwer; 2012.

6. Vanhees L, Lefevre J, Philippaerts R, Martens M, Huygens W, Troosters T, et al. How to assess physical activity? How to assess physical fitness. Eur J Cardiovasc Prev Rehabil. 2005;12: 102-14.

7. Armstrong N, Faukner SG. Aerobic fitness. Paediatr Exerc Physiol. Edinburgh: Churchill Livingstone; 2007. p. 161-87.

8. Mezzani A, Agostoni P, Cohen-Solal A, Corrà U, Jegier A, Kouidi E, et al. Standards for the use of cardiopulmonary exercise testing for the functional evaluation of cardiac patients: a report from the Exercise Physiology Section of the European Association for Cardiovascular Prevention and Rehabilitation. Eur J Cardiovasc Prev Rehabil. 2009;16:249-67.

9. Thompson W, Gordon N, Pescatello L, American College of Sports Medicine, et al. editors. ACSM's guidelines for exercise testing and prescription. 8th ed. New York: Lippincott Williams \& Wilkins; 2010.

10. Rogowski MP, Guilkey JP, Stephens BR, Cole AS, Mahon AD. The influence of maturation on the oxygen uptake efficiency slope. Pediatr Exerc Sci. 2012;24:347-56.

11. Baba R, Nagashima M, Goto M, Nagano Y, Yokota M, Tauchi, et al. Oxygen intake efficiency slope: a new index of cardiorespiratory functional reserve derived from the relationship between oxygen consumption and minute ventilation during incremental exercise. Nagoya J Med Sci. 1996;59:55-62.

12. Baba R, Nagashima M, Nagano Y, Ikoma M, Nishibata K. Role of the oxygen uptake efficiency slope in evaluating exercise tolerance. Arch Dis Child. 1999;81:73-5.

13. Baba R. The oxygen uptake efficiency slope and its value in the assessment of cardiorespiratory functional reserve. Congest Heart Fail. 2000;6:256-8.

14. Akkerman M, Vanbrussel M, Hulzebos HJ, Vanhees L, Helders PJ, Takken T. The oxygen uptake efficiency slope (OUES): what do we know. J Cardiopulm Rehabil Prev. 2010;30:357-573.

15. Buys R, Coeckelberghs E, Vaenhess L, Cornelissen VA. The oxygen uptake efficiency slope in 1411 Caucasian healthy men and women aged 20-60 years: reference values. Eur J Prev Cardiol. 2015;22:356-63.

16. Akkerman M, van Brussel M, Bongers BC, Hulzebos EH, Helders PJ, Takken T. Oxygen uptake efficiency slope in healthy children. Pediatr Exerc. 2010;22:431-41.

17. Gademan MG, Swenne CA, Verwey HF, van de Vooren H, Haest $\mathrm{JC}$, van Exel $\mathrm{HJ}$, et al. Exercise training increases oxygen uptake efficiency slope in chronic heart failure. Eur J Cardiovasc Prev Rehabil. 2008;15:140-4.

18. Gruet M, Brisswalter J, Mely L, Vallier JM. Clinical utility of the oxygen uptake efficiency slope in cystic fibrosis patients. J Cyst Fibros. 2010;9:307-13.

19. Giardini A, Specchia S, Gargiulo G, Sangiorgi D, Picchio FM. Accuracy of oxygen uptake efficiency slope in adults with congenital heart disease. Int J Cardiol. 2009;133:74-9.

20. Marinov B, Kostianev S. Exercise performance and oxygen uptake efficiency slope in obese children performing standardized exercise. Acta Physiol Pharmacol Bulg. 2003;27: $1-6$.

21. Drinkard B, Roberts M, Ranzenhofer LM, Han JC, Yanoff LB, Merke DP, et al. Oxygen uptake efficiency slope as a determinant of fitness in overweight adolescents. Med Sci Sports Exerc. 2007;39:1811-6.
22. Pichon A, Jonville S, Denjean A. Evaluation of the interchange ability of $\mathrm{VO}_{2 \max }$ and oxygen uptake efficiency slope. Can J Appl Physiol. 2007;27:589-601.

23. Sun XG, Hansen JE, Stringer WW. Oxygen uptake efficiency plateau: physiology and reference values. Eur J Appl Physiol. 2012;112:919-28.

24. Kuczmarski RJ, Ogden CL, Guo SS, Grummer-Strawn LM, Flegal KM, Mei Z, et al. 2000 CDC Growth Charts for the United States: methods and development. Vital Health Stat. 2002;246:1-190.

25. Guo SS, Roche AF, Chumlea WC, Johnson C, Kuczmarski RJ, Curtin R. Statistical effects of varying sample sizes on the precision of percentile estimates. Am J Human Biol. 2000;12:64-74.

26. Dezenberg CV, Nagy TR, Gowerl BA, Johnson R, Goran MI. Predicting body composition from anthropometry in pre-adolescent children. Int J Obes. 1999;23:253-9.

27. Slaughter MH, Lohman TG, Boileau RA, Horswill CA, Stillman RJ, Van Loan MD, et al. Skinfold equations for estimation of body fatness in children and youth. Hum Biol. 1988;60:709-23.

28. Myers J, Nieman D. American College of Sports Medicine, editors. ACSM's resources for clinical exercise physiology musculoskeletal, neuromuscular, neoplastic, immunologic, and hematologic conditions. 2nd ed. Wolters Kluwer; 2010.

29. Borg G. A category scale with ratio properties for intermodal and inter individual comparisons. In: Geissler HG, Petzold P, editors. Psychophysical judgment and the process of perception. Berlin: VEB Deutscher Verlag der Wissenschaften; 1982. p. 25-34.

30. James WPT, Research on obesity, editors. Group Report. London: Her Majesty's Stationery Office; 1976.

31. Hallynck TH, Soep H, Thomis JA, Boelaert J, Daneels R, Dettli L. Should clearance be normalised to body surface or to lean body mass. Br J Clin Pharmacol. 1981;11:523-6.

32. 32-Haycock G, Schwartz G, Wisotsky D. Geometric method for measuring body surface area: a height weight formula validated in infants, children and adults. J Pediatr. 1978;93:62-6.

33. Mirwald RL, Baxter-Jones AD, Bailey DA, Beunen GP. An assessment of maturity from anthropometric measurements. Med Sci Sports Exerc. 2002;34:689-94.

34. Hollenberg M, Tager I. Oxygen uptake efficiency slope: an index of exercise performance and cardiopulmonary reserve requiring only submaximal exercise. J Am Coll Cardiol. 2000;36:194-201.

35. Van Laethem C, Bartunek J, Goethals M, Nellens P, Andries E, Vanderheyden M. Oxygen uptake efficiency slope, a new submaximal parameter in evaluating exercise capacity in chronic heart failure patients. Am Heart J. 2005;149:175-80.

36. Van Laethem C, Van De Veire N, De Sutter J, Bartunek J, De Backer G, Goethals M, et al. Prospective evaluation of the oxygen uptake efficiency slope as a submaximal predictor of peak oxygen uptake in aged patients with ischemic heart disease. Am Heart J. 2006;152:9-15.

37. Marinov B, Mandadzhieva S, Kostianev S. Oxygen uptake efficiency slope in healthy 7-18 year-old children. Pediatr Exerc Sci. 2007:159-70.

38. Bongers B, Hulzebos EH, Arets BG, Takken T. Validity of the oxygen uptake efficiency slope in children with cystic fibrosis and mild-to-moderate airflow obstruction. In: Armstrong N, Williams C, editors. Children and exercise XXVII: the proceedings of the XXVIIth International Symposium of the European Group of Pediatric Work Physiology, September 2011.

39. Drinkard B, Roberts MD, Ranzenhofer LM, Han JC, Yanoff LB, Merke DP, et al. Oxygen uptake efficiency slope as a determinant of fitness in overweight adolescents. Med Sci Sports Exerc. 2007;39:1811-6. 